\title{
Modelo de projeção por classe diamétrica para florestas nativas: enfoque na função probabilística de Weibull
}

\author{
Rodrigo Geroni Mendes Nascimento ${ }^{1}$, Sebastião do Amaral Machado ${ }^{1}$, Afonso Figueiredo Filho ${ }^{1}$, Niro Higuchi \\ ${ }^{1}$ Universidade Federal do Paraná, P.O. Box. 19081, CEP 81531-970, Curitiba, PR, Brasil \\ 2Instituto Nacional de Pesquisas da Amazônia, Av. André Araújo, 2936, CEP 69060-001, Manaus , AM, Brasil
}

\author{
"Autor correspondente: \\ geronimendes@hotmail.com \\ Termos para indexação: \\ Modelo implícito de projeção \\ Funções densidade de probabilidade \\ Florestas multiâneas e heterogêneas \\ Index terms: \\ Implicit model of projection \\ Probability density functions \\ Mixed and uneven aged forests
}

\begin{abstract}
Resumo - Em 1979 a técnica de modelagem de distribuições diamétricas por funções probabilísticas foi aplicada pela primeira vez por Hyink \& Moser na prognose do crescimento e da produção de florestas multiâneas e heterogêneas. Entretanto, atualmente, poucos trabalhos a utilizam no planejamento da produção dessas florestas por desconhecerem a viabilidade operacional da técnica. Sendo assim, esse trabalho visa apresentar uma revisão das características que propiciam a modelagem do crescimento e da produção por classe diamétrica, destacando a importância da dinâmica do recrutamento, mortalidade, sobrevivência, bem como dos atributos populacionais correlacionados à modelagem da distribuição de Weibull, apresentando as particularidades estatísticas utilizadas na modelagem da produção por esse método.
\end{abstract}

\section{Projection model by diameter class for native forests: focus on the Weibull probability function}

\section{Introdução}

O manejo florestal é a busca da administração ótima do recurso florestal, visando sempre lucro, renda e oportunidade de trabalho à sociedade, menor impacto possível à natureza e o uso contínuo do recurso evitando sua escassez ou sua degradação. Essa compilação de definições de diferentes autores (Mantel, 1959; Meyer et al., 1961; Richter, 1963; Daniel et al., 1982; Vanclay, 1994, Higuchi et al., 2008; Hosokawa et al., 2008;
Schneider, 2009), atualmente é amplamente difundida na sociedade, porém pouco conhecida ou realmente aplicada no manejo de nossas florestas.

A priori, principalmente para os florestais, não deveria haver distinção entre os termos Manejo Florestal, Manejo Florestal Sustentável, Bom Manejo, Manejo de Impacto Reduzido, entre outros comuns e excessivamente apresentados nos meios de comunicação. Entretanto, o uso da floresta pela sociedade nem sempre é sustentável 
ou visa o menor impacto à natureza, gerando casos que denotam a ausência do conhecimento da ciência florestal, e que incentivam a concepção de novos jargões de sustentabilidade que expressam a mesma ideia do manejo florestal.

Todo gestor de recursos naturais, notadamente aqueles dedicados ao manejo das florestas, deve saber e estar consciente das peculiaridades clássicas da atividade florestal; isto significa que as florestas contém algo mais do que árvores e o seu potencial representa algo mais do que madeira (Berger \& Padilha Junior, 2007). Dessa forma, o conhecimento dos aspectos silviculturais e culturais dos povoamentos florestais, bem como a obtenção das informações concernentes à composição florística e estrutural, são a base do manejo florestal e determinam o acompanhamento da dinâmica do recurso florestal (Hosokawa et al., 2008).

Comumente os profissionais do setor florestal utilizam modelos matemáticos como forma de controle e projeção da estrutura e da produção de povoamentos, utilizando informações correntes para inferir cenários futuros. No geral, esses modelos são representações de qualquer fenômeno no mundo real, sendo uma ferramenta útil na reflexão, prognose e tomada de decisões em horizontes de planejamento de médio e longo prazos (Buongiorno \& Gilles, 1987).

Segundo Peng (2000) o primeiro modelo de crescimento e produção de florestas multiâneas produzido nos moldes similares aos atuais foi o trabalho de Moser \& Hall (1969).

Desde então, muitos modelos foram usados, com metodologias que passaram de uma abordagem empírica para uma mais ecológica, mecanística, baseada em processos que incorporaram várias técnicas, tais como sistemas de equações, projeções por tabela de volume não-linear, cadeia de Markov, modelos matriciais e redes neurais (Peng, 2000).

O destaque entre as técnicas de modelagem são os modelos de projeção por distribuição diamétrica, conhecidos como modelos implícitos de crescimento e produção (Clutter et al., 1983). Essa técnica de modelagem foi empregada pela primeira vez em plantios florestais por Clutter \& Bennett (1965); sendo então difundida e posteriormente empregada na prognose em plantios e florestas. Hyink \& Moser (1979) foram os primeiros pesquisadores a empregarem na modelagem da produção de florestas multiâneas.
A técnica se comprovou eficiente quando aplicada a modelagem de florestas naturais, dando destaque à função densidade de probabilididade de Weibull, que desde 1973, quando foi apresentada (Bailey \& Dell, 1973), é a função probabílistica mais usada na descrição da distribuição diamétrica de plantios florestais e florestas multiâneas e heterogêneas (Clutter et al., 1983; Batista, 1989).

Este trabalho objetiva revisar as características ligadas ao desenvolvimento e aplicação de modelos de crescimento e produção por distribuição diamétrica, revisando os aspectos da modelagem de povoamentos nativos, primários ou que sofreram alterações estruturais, sendo uma alternativa para avaliar e dar embasamento técnico ao manejo florestal que vem sendo realizado nas florestas multiâneas e heterogêneas de nosso país.

\section{Dinâmica Florestal}

Antes que a vegetação de um determinado local alcance uma relativa estabilidade em suas características fisiológicas, estruturais e florísticas, ocorre uma série de mudanças nas comunidades. Este processo é que se denomina de sucessão vegetal ou dinâmica (Odum, 1976). Essa dinâmica estrutural e florística são causadas por uma diversidade de fatores, causando heterogeneidade ambiental no espaço e no tempo numa ampla escala de ação.

A simples queda de um indivíduo arbóreo na floresta proporciona um aumento da complexidade estrutural e da diversidade de espécies, principalmente no local do evento. Outros distúrbios, que operam em outras escalas, como a exploração da floresta e sua fragmentação, os surtos de incêndio, furacões, deslizamentos e enchentes, também são conhecidos por ter um forte efeito sobre a dinâmica florestal e consequentemente sobre a composição e estrutura das árvores e suas comunidades (Johnson \& Miyanishi, 2007).

No âmbito geral, segundo Vanclay (1994), os estudos da dinâmica de populações arbóreas têm como principal objetivo o conhecimento das taxas de mortalidade e recrutamento, as quais, quando analisadas em conjunto com as taxas de crescimento, indicam as alterações demográficas da população e os possíveis fatores que estão influenciando este processo, suas implicações para a comunidade florestal e as possíveis práticas de manejo a serem realizadas na floresta em foco. 


\section{Crescimento e incremento}

O termo crescimento se refere ao aumento das dimensões de um ou mais indivíduos em um povoamento florestal ao longo de um determinado período de tempo, sendo verificado pelo avanço de suas dimensões físicas, ocasionando uma reação à ação das leis naturais, condicionadas ao clima, solo, espécie, composição florística e idade (Campos, 1970; Assmann, 1970; Vanclay, 1994; Schneider, 2009). O resultado das interações de diferentes processos, como a fotossíntese, respiração, relações de água, nutrição mineral, características genéticas, entre outros, determinam o crescimento da planta (Campos, 1970; Lambers et al., 1998).

Em termos de aumento de volume, de peso e de dimensões lineares de unidades estruturais, o crescimento é função do que a planta armazena, ou seja, o que é produzido e acumulado na biomassa da planta (Benincasa, 1988). As taxas de crescimento das árvores são altamente variáveis existindo grandes diferenças entre espécies (Assmann, 1970), bem como entre árvores da mesma espécie, classes de tamanho, constituição genética, ou ainda devido à adaptação a diferentes habitats (Shuggart, 1984).

A quantificação do crescimento de um indivíduo florestal analisado por medições sucessivas separadas por um determinado período de tempo é chamada de incremento (Assmann, 1970). O incremento determina o rendimento e pode ser considerado como a "taxa de acumulação" de um determinado produto; no âmbito das ciências florestais é a taxa de acumulação de rendimento (Synnott, 1978).

A taxa de crescimento é um dos fatores determinantes a ser considerado nos planos de manejo florestal, sendo uma informação básica na estimativa da produção florestal, ordenamento e credibilidade de um plano de manejo sustentável (Silva et al., 2001; Schneider, 2009). Essa taxa determina a viabilidade técnica e econômica de empreendimentos, pois influencia na escolha de diferentes regimes de manejo que definem o horizonte de planejamento, o período de rotação florestal, ou o ciclo de corte em florestas multiâneas (Clutter et al., 1983; Buongiorno \& Gilles, 1987; Berger \& Padilha Junior, 2007).

As características do sítio determinam a dinâmica dos povoamentos florestais, sejam eles multiâneos e heterogêneos em idades e espécies ou plantios homogêneos (Vanclay, 1994), sendo que a taxa de crescimento nos primeiros assume um valor médio dinâmico, em que a floresta oscila em produção, tanto em número de árvores, quanto de espécies e biomassa (Silva et al., 2001; Higuchi et al., 2008). Em florestas perturbadas pela ação humana, ou eventos naturais, a taxa de crescimento é variável no tempo e nos diferentes sítios assumindo uma média dinâmica, como em florestas primárias, após muitos anos de sucessão florestal (Rocha et al., 2003; Silva et al., 2001; Tsuchiya et al., 2006).

\section{Mortalidade e sobrevivência}

A mortalidade pode ser definida como o número de indivíduos que morrem em um dado período no tempo, podendo ser expressa como uma taxa explicitada pela razão entre o número de indivíduos que morreram e o total existente anteriormente ao evento causador da morte (Clutter et al., 1983; Vanclay, 1994). Diversos fatores podem causar a mortalidade em povoamentos florestais, tais como a idade ou senilidade, competição e supressão, doenças e pragas, alterações climáticas, fogos silvestres, tempestades convectivas, anelamento e envenenamento, injúrias, corte ou abate de árvore (Vanclay, 1994; Sanquetta, 1996; Rossi et al., 2007; Marra, 2010).

O estudo dos processos de recrutamento e mortalidade em sistemas dinâmicos como florestas tropicais são imprescindíveis para explicar a dinâmica, composição e estrutura da floresta (Bugmann, 2001). O conhecimento das taxas de mortalidade florestal, em todas as escalas, é necessário para avançar no entendimento da fitogeografia e dos sistemas naturais (Rossi et al., 2007, Pinto, 2008).

A mortalidade e a reprodução constituem juntas num ponto de partida para a maioria dos estudos em dinâmica de populações (Solomom, 1980). Muitos modelos de crescimento para plantios florestais evitam o problema de predizer a mortalidade, assumindo que ela não ocorre em povoamentos bem manejados. Entretanto, esse pressuposto é inapropriado quando o objeto de estudo é uma floresta natural onde a mortalidade é significativa e afeta a produção (Vanclay, 1994).

Todo evento que afeta a estrutura da população florestal, seja ele regular ou irregular no tempo, causando a redução do número de árvores, são "inputs" da dinâmica florestal (Rossi et al., 2007). Segundo Moser (1972), a mortalidade em povoamentos inequiâneos é largamente dependente da estrutura do povoamento, sendo essa variável expressa em área basal e número de 
árvores por hectare, ou qualquer índice de competição que possa indicar a sobrevivência futura ou a necessidade de futuras intervenções silviculturais no povoamento (Clutter et al., 1983).

Essa competição é mais acirrada nas classes diamétricas de menores tamanhos, devido à forma da distribuição de frequências em povoamentos multiâneos, em que a densidade em termos de indivíduos é maior nas primeiras classes (Rossi et al., 2007). O recrutamento é dependente da mortalidade, e por sua vez a mortalidade regular aumenta conforme o ingresso atinge a capacidade limite do sítio, sendo a regeneração florestal, junto à mortalidade, os agentes causadores da competição e renovação da floresta em um ciclo dinâmico.

A mortalidade, por ser um evento aleatório e dinâmico em florestas naturais, só pode ser mensurada por meio de parcelas permanentes representativas da floresta (Clutter et al., 1983; Bugmann, 2001; Rossi et al., 2007). O crescimento dos atributos do povoamento florestal são sempre cumulativos e contínuos no tempo, já a mortalidade é um evento totalmente aleatório, descontínuo e sua medição precisa só é possível pelo acompanhamento da floresta por vários anos.

O contrário da mortalidade é a sobrevivência dos indivíduos na floresta, sendo um evento cumulativo, reflexo do balanço entre a mortalidade e o recrutamento, indicativo de competição e produção florestal em termos de número de indivíduos por unidade de área. É uma variável facilmente mensurável na floresta, pois necessita apenas da contagem dos indivíduos vivos por unidade de área.

Modelar o número de árvores por unidade de área em florestas multiâneas deveria adotar como variáveis explicativas as taxas de recrutamento e mortalidade, pois a interação desses processos com as características do sitio condicionam a sobrevivência das árvores nas diferentes classes diamétricas e fisionomias florestais. Muitos modelos de crescimento para florestas equiâneas predizem a mortalidade como função resposta da densidade, assumindo que há uma relação simples entre a densidade máxima do povoamento e o diâmetro médio das árvores (Vanclay, 1994).

Entretanto, muitos modelos foram criados para expressar a sobrevivência, tanto para plantios como para florestas multiâneas e heterogêneas, utilizando a idade e sítio como variáveis independentes na concepção de modelos destinados a plantios (Clutter et al., 1983; Vanclay, 1994; Rossi et al., 2007), e variáveis ligadas aos atributos da população, como área basal, números de indivíduos em determinados grupos ecológicos ou números de espécies comerciais, diâmetro médio ou somatório dos diâmetros, na modelagem de florestas multiâneas (Moser, 1972; Vanclay, 1994; Bugmann, 2001; Rossi et al., 2007).

A mortalidade é um evento de grande importância sobre a produção florestal, que se manifesta, sobretudo, na confecção de tabelas de produção para povoamentos desbastados (Clutter et al., 1983; Schneider, 2009) ou explorados seletivamente, sendo portanto um tópico básico na modelagem do crescimento e produção de povoamentos.

\section{Recrutamento}

Também conhecido como ingresso, o recrutamento é o número de árvores que atinge o diâmetro mínimo considerado no monitoramento da floresta (Pinto, 2008). Árvores recrutadas, portanto, são aquelas que ingressaram em um levantamento, mas não estavam presentes anteriormente ou, são aquelas árvores que ultrapassaram a dimensão mínima estabelecida para a inclusão no intervalo entre dois tempos (Vanclay, 1994; Silva et al., 2001).

O recrutamento é obtido pelo uso de parcelas permanentes convencionais (Silva et al., 2001; Tsuchiya et al., 2006), e sua dinâmica esta relacionada à densidade do povoamento, mortalidade de árvores, histórico da exploração, tipo de floresta, presença de árvores porta-sementes, dinâmica de clareiras, e outros fatores empiricamente determináveis na parcela (Tsuchiya et al., 2006; Pinto, 2008). Segundo Gomide (1997), o estudo do ingresso em florestas tropicais úmidas tem grande importância do ponto de vista silvicultural, assim como sua quantidade e qualidade determinam com que sucesso a floresta está sendo "alimentada" com plântulas e pequenas árvores de espécies comerciais.

A resposta aos distúrbios que causam a mortalidade dos indivíduos na floresta primária é o recrutamento, sendo esses ocasionados por fatores bióticos, abióticos ou antrópicos (Tabarelli \& Mantovani, 1997; Wright et al., 2003). A sucessão florestal pode ser acompanhada pelos estudos ligados a recrutamento e mortalidade da floresta, pois há uma dinâmica desigual entre os grupos ecológicos nesse processo, tanto em épocas como intensidade de ocorrência.

O estudo da taxa de recrutamento está diretamente relacionado ao acompanhamento da regeneração das espécies de interesse nos planos de manejo florestal 
e/ou com fins de entendimento de distúrbios naturais (Hosokawa et al., 2008; Marra, 2010). No sistema de manejo de corte seletivo, a avaliação da regeneração de espécies de valor comercial, bem como o seu ingresso em classes diamétricas comerciais, é de suma importância, já que é um indício da viabilidade técnica e econômica do regime de manejo empregado (Hosokawa et al., 2008).

Modelos de projeção da produção de florestas multiâneas e heterogêneas devem mensurar o efeito do recrutamento e da mortalidade na produção futura, já que a inclusão dessas contribui em maior eficiência das estimativas biométricas (Clutter et al., 1983; Vanclay, 1994). Esses modelos, quando aplicados em florestas secundárias, devem ter características matemáticas que evidenciem o crescimento no número de indivíduos, tais como modelos biomatemáticos ou equações diferenciais como proposto por Moser (1972), pois tanto o recrutamento como a mortalidade são eventos cumulativos no tempo e apresentam taxas diferenciadas ao longo da sucessão florestal.

\section{Predição e projeção do crescimento e produção}

Em florestas multiâneas e heterogêneas a modelagem do crescimento e produção são feitas com fins de predição e projeção dos atributos globais do povoamento bem como da distribuição diamétrica da floresta (Vanclay, 1994). Essa técnica é amplamente utilizada no manejo de povoamentos florestais e é de suma importância no planejamento da empresa florestal, pois possibilita ao gestor simular cenários de exploração e colheita, bem como de análise de investimentos futuros ligados a aquisição de recursos e contratação de mão de obra (Buongiorno \& Gilles, 1987).

Para a prognose da estrutura e da produção futura da floresta, a inclusão das variáveis idade e sítio são primordiais (Clutter et al., 1983), já que a definição implícita do crescimento e da produção é o tempo, que expressa a mudança da produção em determinada "idade" do povoamento, sendo alterada por características únicas do meio em que vivem, ou seja, seu sítio.

A idade é a primeira variável explicativa nos estudos de crescimento e produção de povoamentos equiâneos (Sanquetta, 1996). Já a qualidade de sítio, quando incluída no ajuste dos modelos de crescimento e produção, beneficia as estimativas biométricas em termos de precisão e possibilita a prognose em diferentes densidades do povoamento (Clutter et al. 1983).
Em florestas secundárias o tempo decorrente após uma perturbação no dossel, ou idade da perturbação, é uma variável comumente usada na modelagem da dinâmica de sucessão de clareiras (Shuggart, 1984; Vanclay, 1994; Bugmann, 2001). Semelhante a um povoamento equiâneo, em que um estoque de árvores remanescentes permanece após o desbaste, as árvores remanescentes a uma exploração num povoamento florestal inequiâneo são referidas como estoque de crescimento (Clutter et al., 1983).

As modificações estruturais causadas pela mortalidade alteram a composição florestal, bem como as características do sítio onde ocorreu o evento (Shuggart, 1984), sendo que a velocidade de fechamento de clareiras, a variação diamétrica e estrutural dessas áreas perturbadas, são indicativos da qualidade do sítio (Grubb, 1977), podendo ser incluídas em modelos de crescimento e produção por distribuição diamétrica.

Dessa forma, ao compor um modelo que descreva o crescimento e a produção futura de florestas multiâneas por classe diamétrica, necessariamente deve-se analisar todos os aspectos que influenciam a dinâmica populacional em termos de recrutamento, mortalidade, grau de ocupação e competição, sítio e idade, bem como o efeito desses fatores sobre o ingresso entre as classes diamétricas ao longo da distribuição de frequência da floresta.

\section{Modelos de projeção por classe diamétrica}

Modelos de crescimento e produção por distribuição diamétrica, ou modelos implícitos de produção, estimam o número de árvores por hectare por classe de diâmetros nas idades presente e futura (Clutter et al., 1983; Vanclay, 1994), e a partir de seu emprego associado à uma equação de volume, de afilamento ou de razão volumétrica, pode-se estimar a produção por classe, sendo úteis no planejamento da produção quando há interesse em multiprodutos da madeira (Sanquetta, 1996).

Dentre os modelos por classe diamétrica mais aplicados em florestas multiâneas e heterogêneas, a cadeia de Markov se consagra pela fácil aplicabilidade e menor quantidade de medições necessárias para efetuar a prognose (Vanclay, 1994; Higuchi et al., 2008). Entretanto, um aspecto questionável é a suposição de que as probabilidades, uma vez calculadas e inseridas na matriz de transição, permanecerão constantes ao longo da vida da floresta (Arce et al., 1997), sendo esses valores dependentes do intervalo de classe e de tempo adotados. 
Contornando os problemas ligados a Cadeia de Markov, Hyink \& Moser (1979) aplicaram pela primeira vez em florestas multiâneas a técnica desenvolvida por Clutter \& Bennett (1965). Essa técnica é conhecida pela necessidade de recuperação dos coeficientes de funções probabilísticas a partir de momentos amostrais estimados para um ano futuro ou presente, visando à estimativa da distribuição diamétrica da floresta em análise.

Para efetuar a prognose da produção a partir de um modelo implícito de produção é necessário efetuar os seguintes passos: obter dados de parcelas permanentes, de maneira representativa; estimar os coeficientes da função densidade de probabilidade associada a cada parcela amostrada do povoamento; obter equações de regressão para estimar os parâmetros da função probabilística, ou os momentos, em uma segunda ocasião, utilizando como variáveis explicativas os atributos da floresta; e avaliar e aplicar o modelo (Scolforo, 2006).

Qualquer método de distribuição diamétrica prevê a distribuição de frequência em diâmetro e alturas por classe de diâmetro a partir de certos atributos estatísticos do povoamento, por exemplo, idade, índice de sítio e densidade e altura média das dominantes e co-dominantes (Clutter et al., 1983; Sanquetta, 1996). As funções densidade de probabilidade são atualmente as principais ferramentas na modelagem estrutural de plantios florestais e florestas multiâneas e heterogêneas, sendo o quesito base de um modelo por classe diamétrica.

\section{Distribuição diamétrica e funções densidade de probabilidade}

Por meio da distribuição de uma variável dendrométrica, como o diâmetro à altura do peito (DAP), diâmetro de copa, altura total, área transversal, volume individual, dentre outras, é possível descrever a floresta ou uma espécie que a compõe, pelo seu grau de ocupação, dominância, maior ou menor número de indivíduos por classe, maior concentração de volume por classe ou sucessão da espécie.

Segundo Arce (2004), na avaliação da estrutura horizontal, a distribuição diamétrica é a ferramenta mais simples para caracterizar a estrutura da floresta. Com o conhecimento da estrutura diamétrica, a prescrição de intervenções no manejo e determinação do rendimento da floresta por tipo de produto, se torna mais fácil e fundamental (Prodan et al., 1997).

Em florestas multiâneas, o tamanho das árvores pode ser uma característica mais importante que a idade, sendo a distribuição diamétrica um informativo ecológico mais adequado na ausência da idade (Enright \& Ogden, 1979). Em florestas nativas a distribuição diamétrica é importante, pois mostra a amplitude dos diâmetros, onde acontece maior concentração do número de árvores, servindo para distinguir diferentes tipos florestais, grau de ocupação dos indivíduos e elaboração de tabelas de produção que consideram a dinâmica da população florestal (Scolforo, 2006).

Barros et al., (1979) afirmaram que desde o trabalho de De Liocurt (1898) ${ }^{1}$ vários modelos matemáticos, denominados funções densidade de probabilidade (fdps), são usados na ciência florestal por serem capazes de descrever a estrutura diamétrica de plantios e demais populações florestais. $\mathrm{Na}$ distribuição diamétrica de florestas têm sido usadas funções probabilísticas muito conhecidas por apresentarem, de forma mais clara, o comportamento estrutural da floresta e auxiliar na prognose de plantios florestais, dentre elas a função Beta, Exponencial, Gamma, Normal, Lognormal, SB de Johnson, Weibull 2 e 3 parâmetros, entre outras.

Em geral, o uso dessas funções está diretamente ligado à natureza dos dados a que ela se relaciona (Marques, 2003). No Brasil as funções densidade de probabilidade foram utilizadas pioneiramente por Barros et al., (1979), na descrição da estrutura diamétrica da floresta amazônica, sendo a técnica amplamente divulgada na região por inúmeros trabalhos, tais como Hosokawa (1981), Higuchi (1988), Umaña (1998), Higuchi (2007), para a modelagem diamétrica de trabalhos realizados na região.

Algumas funções probabilísticas possuem grande capacidade estimativa da variável de interesse usando uma pequena quantidade de dados, outras requerem grande série de observações (Krishnamoorthy, 2006; Walck, 2007). Entretanto, sabe-se que variáveis contínuas possuem características diferentes, mesmo tendo um grau de dependência uma com as outras, como é o caso das variáveis dendrométricas, exigindo assim particularidades em seu ajuste, conforme a função probabilística utilizada.

A eficiência do modelo quando distribuído em um histograma de frequência se dá pelos testes de aderência de Kolmogorov - Smirnov (K-S) e Qui-quadrado $\left(\chi^{2}\right)$ (Scolforo, 2006; Higuchi et al., 2008; Orellana, 2009). O primeiro é definido como a diferença máxima absoluta entre a frequência observada acumulada e a frequência esperada acumulada (Scolforo, 2006). O segundo

${ }^{1}$ De Liocourt, F. De l'amenagement des sapinières. Besançon: Société forestière de Franche-Comté et Belfort, 1898. p. 396-409. (Bulletin trimestriel). 
compara a distribuição de frequência observada com a estimada, a fim de avaliar se as proporções observadas destes eventos mostram ou não diferenças significativas ou se as amostras diferem significativamente quanto às proporções desses acontecimentos (Marques, 2003).

Os testes de aderência K-S e $\chi^{2}$ testam as hipóteses $\mathrm{H}_{0}$ de que os diâmetros observados seguem as distribuições propostas pela fdp ajustada, conforme as diferentes formas de cálculo apresentadas:

- Teste de Kolmogorov - Smirnov:

$$
D=M A X_{X} \mid F o_{x}-F e_{x}
$$

Em que:

$\boldsymbol{F o}_{x}$ é a frequência observada acumulada;

$\boldsymbol{F e}_{x}$ é a frequência esperada acumulada;

$\boldsymbol{D}$ é o ponto de máxima divergência entre as frequências.

- $\quad$ Teste de $\chi^{2}$

(2) $\chi_{v}^{2}=\sum_{i=1}^{n} \frac{\left(o_{i}-e_{i}\right)^{2}}{e_{i}}$

(3) $\quad v=k-1-m$

Em que:

$\boldsymbol{o}_{i}$ é a frequência observada;

$\boldsymbol{e}_{i}$ é a frequência esperada ou teórica, de acordo com o modelo testado;

$v$ é o grau de liberdade do teste;

$\boldsymbol{k}$ é o número de classes ou valores considerados e;

$\boldsymbol{m}$ é o número de coeficientes do modelo teórico.

A flexibilidade dessas funções permite seu uso em diferentes situações nas grandes áreas da ciência, como engenharias, ciências biológicas, humanas, sociais, etc. (Krishnamoorthy, 2006; Walck, 2007). Nas ciências florestais as funções densidade de probabilidade já foram empregadas na descrição da distribuição hipsométrica, distribuição de precipitação de chuva, modelos de sobrevivência, modelos de classificação de sitio e principalmente distribuição diamétrica (Weibull, 1951; Singh, 1987; Zeide, 1993; Scolforo, 2006; Machado et al., 2010) dando destaque à função concebida por Woloddi Weibull, em 1939.

\section{Distribuição probabilística de Weibull}

A origem da função de Weibull se deu pelo trabalho de Weibull (1939), cujo objetivo era analisar valores de resistência máxima de materiais, por meio de um modelo probabilístico que pudesse descrever resultados discrepantes da teoria clássica do tema. Em 1951, devido à ampla divulgação do modelo no período de guerra e pós-guerra, Weibull apresentou a grande aplicabilidade do modelo na descrição de diferentes eventos, utilizando exemplos de seu funcionamento bem como sua forma de ajuste.

Na ciência florestal, a introdução da função de distribuição Weibull aos problemas relacionados à silvicultura e manejo florestal é atribuída a Bailey e Dell no ano de 1973 (Prodan et al., 1997; Scolforo, 2006; Higuchi, 2007). Desde então, esta distribuição tem sido amplamente utilizada para descrever a distribuição de diâmetros, tanto em povoamentos equiâneos como inequiâneos, especialmente nos Estados Unidos (Scolforo, 2006).

O modelo possui características matemáticas que o fazem ser um ferramental descritivo muito flexível, podendo assumir, conforme os valores de seus coeficientes, várias formas e assimetrias, o que possibilita seu emprego na descrição de diferentes relações dendrométricas ou tipologias florestais. Sua aplicabilidade é preferida nas ciências florestais devido à grande flexibilidade, precisão e correlação existente entre os seus coeficientes e os atributos populacionais da floresta ou do plantio florestal. A função densidade de probabilidade de Weibull, bem como sua forma acumulada, pode ser representada como segue:

(4) $f(x)=\left(\frac{c}{b}\right)\left(\frac{x-a}{b}\right)^{c-1} e^{-\left(\frac{x-a}{b}\right)^{c}}$

(5)

$$
F(x)=1-e^{-\left(\frac{x-a}{b}\right)^{c}}
$$

Em que:

$\boldsymbol{x}$ é a variável de interesse;

$\boldsymbol{f}(\boldsymbol{x})$ é a função densidade de probabilidade de Weibull;

$\boldsymbol{F}(\boldsymbol{x})$ é a função densidade acumulada de Weibull;

$\boldsymbol{a}$ é o coeficiente de alocação do modelo;

$\boldsymbol{b}$ é o coeficiente da função referente à escala e;

$c$ é o coeficiente que determina a forma da curva. 
O coeficiente $\boldsymbol{a}$ do modelo quando igual a zero denota uma particularidade da função Weibull, conhecida como Weibull 2 parâmetros, ou alocada na origem (Figura, 2010). Esse coeficiente pode assumir o valor mínimo do rol de dados a que se propõe a modelagem, sendo este valor sempre positivo, menor ou igual ao menor diâmetro do povoamento. Assim, o valor de $\boldsymbol{a}$ deve estar sempre compreendido entre zero e o $\boldsymbol{d}_{\min }$ (Knoebel et al., 1988).

Dado uma forma e uma alocação fixas sobre o eixo das abscissas, a variação do valor do coeficiente $\boldsymbol{b}$ determina a amplitude de variação entre o mínimo e máximo da função, modificando a escala em termos de curtose (Marques, 2003; Krishnamoorthy, 2006; Walck, 2007). Quanto menor o valor de $\boldsymbol{b}$, mais leptocúrtica a distribuição se apresenta; quanto maior o valor de $\boldsymbol{b}$, mais platicúrtica será a distribuição. Essa ultima informação é muito conveniente no ajuste da Weibull na descrição da distribuição diamétrica das espécies pioneiras em florestas tropicais (Nascimento, 2012), pois o $\boldsymbol{b}$ é um valor diretamente proporcional a moda quando $c \geq 1$ no ajuste da distribuição (Tabela 1 ).

A função probabilística Weibull pode ter um grande número de formas todas definidas pelo coeficiente $\boldsymbol{c}$, pois quando $c<1$, a função é decrescente de maneira abrupta; quando $c=1$ a função é exponencial negativa; para valores onde $\boldsymbol{c}>1$ a função é unimodal; quando $1<\boldsymbol{c}<$ 3,6 a distribuição tem assimetria positiva; quando $c=3,6$ é aproximadamente uma distribuição normal e quando $c$ $>$ 3,6 a distribuição tem assimetria negativa (Baker et al., 2005; Scolforo, 2006; Figura, 2010).

Existem diferentes formas de ajustar a distribuição Weibull, podendo-se utilizar os métodos da máxima verossimilhança, método dos momentos, método dos percentis, simulated annealing, redes neurais artificiais, regressão linear e não-linear, método gráfico e aproximação linear (Cao, 2004; Scolforo, 2006; Campos \& Leite, 2009; Figura, 2010). Desses métodos se destacam os métodos da máxima verossimilhança, momentos e regressão não linear, apresentando sempre bons resultados em diferentes trabalhos (Batista, 1989; Orellana, 2009; Figura, 2010).

O método dos momentos é bastante difundido no meio florestal, devido à correlação entre os coeficientes de escala e forma com os momentos amostrais de $1^{\circ}$ à $12^{\circ}$ ordem centrados na origem (Krishnamoorthy, 2006). Dos momentos apresentados, todos são possíveis de serem determinados a partir da função Weibull ajustada, porém os valores apresentados são apenas para o caso particular da fdp onde $\boldsymbol{a}=0$.
Tabela 1. Momentos de $1^{\circ}$ À $12^{\circ}$ ordem da função densidade de probabilidade de Weibull, quando $\boldsymbol{a}=0$ ou $\boldsymbol{y}=\boldsymbol{x}-\boldsymbol{a}$

\begin{tabular}{|c|c|}
\hline Momentos amostrais & Forma de concepção \\
\hline Média & $\bar{x}=b \Gamma_{1}$ \\
\hline Variância & $\sigma^{2}=b^{2}\left[\Gamma_{2}-\Gamma_{1}^{2}\right]$ \\
\hline Moda & $x_{\text {mod }}=b\left(1-\frac{1}{c}\right)^{\frac{1}{c}}$, quando $c \geq 1$ \\
\hline Mediana & $x_{m e d}=b[\operatorname{Ln}(2)]^{\frac{1}{c}}$ \\
\hline Coeficiente de Variação & $C V=\frac{\sqrt{\Gamma_{2}-\Gamma_{1}^{2}}}{\Gamma_{1}}$ \\
\hline Coeficiente de Assimetria & $\sigma^{3}=\frac{\Gamma_{3}-3 \Gamma_{1} \cdot \Gamma_{2}+2 \Gamma_{1}^{3}}{\left[\Gamma_{2}-\Gamma_{1}^{2}\right]^{\frac{3}{2}}}$ \\
\hline Momentos sobre a origem & $E\left(x^{k}\right)=b^{k} \Gamma_{k}$ \\
\hline $\begin{array}{l}\text { Função de distribuição } \\
\text { Inversa }(p)\end{array}$ & $x_{p}=b[-\operatorname{Ln}(1-p)]^{1}$ \\
\hline Função de sobrevivência & $P(X>x)=e^{-\left(\frac{x}{b}\right)^{c}}$ \\
\hline $\begin{array}{l}\text { Função de sobrevivência } \\
\text { inversa }(p)\end{array}$ & $x_{p}=b\left[\left(\frac{1}{c}\right) \cdot \operatorname{Ln}(-p)\right]$ \\
\hline Taxa de risco & $i_{x}=\frac{c \cdot x^{c-1}}{b^{c}}$ \\
\hline Função de risco & $f\left(x_{i}\right)=\left(\frac{x}{b}\right)^{c}$ \\
\hline
\end{tabular}

Legenda: $\Gamma \mathrm{k}=\Gamma(1+\mathrm{k} / \mathrm{c})$, onde k é igual a 1, 2, 3, etc.Fonte: Weibull (1951); Krishnamoorthy (2006).

A maioria dos atributos populacionais de um povoamento florestal é correlacionada com os momentos da média. Qualquer relação estatística pode estimar esses atributos de forma independente aos momentos amostrais de uma função probabilística (Scolforo, 2006; Campos \& Leite, 2009). Essas estimativas, correlacionadas com os momentos amostrais, permitem, por processo iterativo, a recuperação dos coeficientes do modelo probabilístico desde que haja um número maior ou igual de regressões ajustadas para a mesma quantidade de coeficientes a serem estimados da função (Figura, 2010).

Diferentes atributos, tais como coeficiente de variação dos diâmetros, diâmetro médio, diâmetro quadrático médio, sobrevivência (mortalidade e recrutamento), área basal, índice de sito, são usados na recuperação dos coeficientes das funções probabilísticas destinadas a modelagem da produção florestal. Para isso diversos 
modelos são testados e comumente apresentados em diversos trabalhos (Vanclay, 1994; Scolforo, 2006; Rossi et al., 2007; Campos \& Leite, 2009).

\section{Conclusões}

Os tópicos apresentados dão uma orientação no desenvolvimento de modelos de crescimento e produção por classe diamétrica, utilizando a técnica de recuperação dos coeficientes de uma função densidade de probabilidade previamente escolhida para modelagem de florestas mistas em idade e espécies, que sofreram ou não perturbações.

A função de Weibull é o modelo abordado devido à sua grande flexibilidade e variedade de métodos de ajuste que possui, permitindo a estimativa de grande quantidade de momentos da média, esses apropriados na abordagem de diferentes situações possíveis. Devido à sua grande flexibilidade e alternativas de ajuste essa função vem se mostrando como a mais adequada na modelagem de diferentes grupos ecológicos que compõem a floresta tendo êxito em quase toda forma de distribuição.

Os modelos implícitos de projeção desenvolvidos a partir de funções probabilísticas são uma alternativa no controle e gestão bem como na caracterização da dinâmica de florestas tropicais. Entretanto, devido às peculiaridades que cada sítio apresenta sua eficiência no ajuste, ou método de modelagem, sempre estará em função das necessidades do gestor florestal, já que é uma importante técnica em desenvolvimento na ciência florestal.

\section{Referências}

ARCE, J. E. Modelagem da estrutura de florestas clonais de Populus deltoides March através de distribuições diamétricas probabilísticas. Ciência Florestal, Santa Maria, RS, v. 14, n. 1, p. 149-164, 2004.

ARCE, J. E.; PIZATTO, W.; SANQUETTA, C. R.; WENDLING, J. L. G.; MAESTRI, R. Utilização das matrizes de transição na avaliação e simulação precoces do crescimento de povoamentos de Pinus taeda L. Floresta, Curitiba, v. 27, n. 1-2, p. 83-98, 1997.

ASSMANN, E. The principles of forest yield study: studies in the organic production, structure, increment and yield of forest stands. New York: Pergamon Press, 1970. 506 p.

BAILEY, R. L.; DELL, J. R. Quantifying diameter distributions with the Weibull function. Forest Science, Bethesda, v. 19, n. 2, p. 97-104, 1973.

BAKER, J. P.; BUNYAVEJCHEWIN, S.; OLIVER, C. D.; ASHTON, P. S. Disturbance history and historical stand dynamics of a seasonal tropical forest in Western Thailand. Ecological Monographs, Lawrence, v. 3, n. 75, p. 317-343, 2005.
BARROS, P. L. C. de; MACHADO, S. do A.; BURGER, D.; SIQUEIRA, J. D. P. Comparação de modelos descritivos da distribuição diamétrica em uma floresta tropical. Floresta, Curitiba, v. 10, n. 2, p. 19-32, 1979.

BATISTA, J. L. F. A função Weibull como modelo para a distribuição de diâmetros de espécies arbóreas tropicais. 1989. 136 f. Dissertação (Mestrado em Ciências florestais) Escola Superior de Agricultura Luiz de Queiroz, São Paulo, SP.

BENINCASA, M. N. P. Análise de crescimento de plantas: noções básicas. Jaboticabal: FINEP, 1988. 42 p.

BERGER, R.; PADILHA JUNIOR, J. B. Economia florestal. Curitiba, 2007. 143 p.

BUONGIORNO, J.; GILLESS, J. K. Forest management and economics. New York: Macmillan Publishing, 1987. 285 p.

BUGMANN, H. A review of forest gap models. Climatic change, Netherlands, v. 51, p. 259-305, 2001.

CAMPOS, J. C. C.; LEITE, H. G. Mensuração florestal: perguntas e respostas. 3 ed. Viçosa, MG: UFV, 2009. 548 p.

CAO, Q. V. Predicting parameters of a Weibull function for modeling diameter distribution. Forest Science, Lawrence, v. 50, n. 5, p. 682-685, 2004.

CLUTTER, J. L.; BENETT, F. A. Adiameter distributions in old field slash pine plantations. Georgia Forest Research Council Report, Macon, GA, n. 13, p. 1-9, 1965.

CLUTTER, J.; FORTSON, J. C.; PIENAAR, L. V.; BRISTER, G. H.; BAILEY, R. L. Timber management: a quantitative approach. New York: John Wiley e Sons, 1983. 333 p.

DANIEL, P. W.; HELMS, U. E.; BAKER, F. S. Princípios de silvicultura. México: McGraw Hill, 1982. 492 p.

ENRIGHT, N.; OGDEN, J. Applications of transition matrix models in forest dynamics: Araucaria in Papua New Guinea and Nothofagus in New Zealand. Australian Journal of Ecology, Carlton, v. 4, p. 3-23, 1979.

FIGURA, M. A. A distribuição de Weibull na descrição da estrutura diamétrica de Eucalyptus grandis: um enfoque sobre o método dos momentos. 2010. 113 f. Dissertação (Mestrado em Engenharia Florestal) - Universidade Federal do Paraná, Curitiba.

GOMIDE, G. L. A. Estrutura e dinâmica de crescimento de florestas tropicais primária e secundária no estado do Amapá. 1997. 179 f. Dissertação (Mestrado em Engenharia Florestal) - Universidade Federal do Paraná. Curitiba.

GRUBB, P. J. The maintenance of species-richness in plant communities: the importance of the regeneration niche. Biological Reviews of the Cambridge Philosophical Society, Cambridge, UK, v. 52, p. 107-145, 1977.

HIGUCHI, F. G. A influência do tamanho da parcela na precisão da função de distribuição de diâmetro de Weibull da Floresta Primária da Amazônia central. 2007. 59 f. Dissertação (Mestrado em Engenharia Florestal) - Universidade Federal do Paraná. Curitiba. 
HIGUCHI, N.; SANTOS, J.; VIEIRA, G.; RIBEIRO, R. J.; SAKURAI, S. ISHIZUKA, M. SAKAI, T.; TANAKA, N.; SAITO, $\mathrm{S}$. Análise estrutural da floresta primária da bacia do rio cueiras, ZF-2, Manaus-AM, Brasil. Manaus, Amazonas. In: HIGUCHI, N.; CAMPOS, M. A. A.; SAMPAIO, P. T. B.; SANTOS, J. dos. (Ed.). Pesquisas florestais para a conservação da floresta e reabilitação de áreas degradadas da Amazônia. Manaus: INPA/JICA, 1998. p. 52-81.

HIGUCHI, N.; SANTOS, J. dos; SILVA, R. P. da; LIMA, A. N.; TEIXEIRA, L. M.; CARNEIRO, V. M. C.; FELSEMBURGH, C. A.; TRIBUZY, E. S. Noções básicas sobre manejo florestal. 2008. 270 p. Grupo de pesquisas em manejo florestal/INPA. (Apostila).

HOSOKAWA, R. T. Manejo de florestas tropicais úmidas em regime de rendimento sustentado. Curitiba: UFPR, 1981. 125 p. Relatório técnico.

HOSOKAWA, R. T.; MOURA, J. B. de; CUNHA, U. S. da; Introdução ao manejo e economia de florestas. Curitiba: UFPR, 2008. $164 \mathrm{p}$.

HYINK, D. M.; MOSER, J. W. Application of diameter distributions for yield projection in uneven-aged forests. In: FRAYER, W. E. (Ed.). Forest resource inventories: proceedings of a workshop. Fort Collins: Colorado State University, 1979. P. 906-916.

JOHNSON, E. A.; MIYANISHI, K. Plant disturbance ecology: the process and the response. Oxford, UK. Elsevier, 2007. 720 p.

KRISHNAMOORTHY, K. Handbook of statistical distributions with applications. Boca Raton: Chapman \& Hall/CRC, 2006. 344 p.

LAMBERS, H.; CHAPIM III, F. S.; PONS, T. L. Plant physiological ecology. New York: Spring-Verlag, 1998. 299 p.

MACHADO, S. do A.; NASCIMENTO, R. G. M.; MIGUEL, E. P.; TÉO, S. J.; AUGUSTYNCZIK, A. L. D. Distribution of total height, transverse area and volume for Araucaria angustifolia (Bert.) O. Kuntze. Cerne, Lavras, MG, v. 16, n. 1, p. 12-21, jan./mar. 2010.

MANTEL, W. Forsteinrichtung. Frankfurt: Sauerland's Verlag, 1959. $262 \mathrm{p}$.

MARQUES, J. M. Estatística: cursos de engenharia. Curitiba: UFPR, 2003. 191 p. Apostila.

MARRA, D. M. Sucessão florestal em área atingida por tempestade convectiva na Região de Manaus, Amazônia Central. 2010. 106 f. Dissertação (Mestrado em Ciências de Florestas Tropicais) - Instituto Nacional de Pesquisas da Amazônia, Manaus.

MEYER, H. A.; RECKNAGEL, A. B.; STEVENSON, D. D.; BARTOO, R. A. Forest Management. 2nd. ed. New York: The Ronald Press Company, 1961. 282 p.

MOSER, J. W.; HALL, O. F. Deriving growth and yield functions for uneven-aged forest stands. Forest Science, Bethesda, v. 15, n. 1, p. 183-188, 1969.

MOSER, J. W. Dynamics of an uneven-aged forest stand. Forest Science, Bethesda, v. 18, n. 3, p. 184-191, 1972.

NASCIMENTO, R. G. M. Modelagem e prognose da produção de uma floresta tropical úmida densa de terra-firme na Amazônia Central. 2012. 173 f. Dissertação (Mestrado em Ciências Florestais) - Universidade Federal do Paraná, Curitiba.
ODUM, E. P. Fundamentos da ecologia. 2 ed. Lisboa: Fundação Calouste Gulbenkian, 1976. 603 p.

ORELLANA, E. Funções densidade de probabilidade no ajuste da distribuição diamétrica de um fragmento de floresta ombrófila mista. 2009. 139 f. Dissertação (Mestrado em Ciências Florestais) - Universidade Estadual do Centro Oeste, Irati.

PENG, C. Growth and yield models for uneven-aged stands: past, present and future. Forest Ecology and Management, Amsterdam, v. 132, n. 1-3, p. 259-279, 2000.

PINTO, A. C. M. Dinâmica de uma floresta de terra firme manejada experimentalmente na região de Manaus (AM). 2008. 167 f. Tese (Doutorado em Ciências Florestais Tropicais) Instituto Nacional de Pesquisas da Amazônia/Universidade Federal do Amazonas, Manaus.

PRODAN, M.; PETERS, R.; COX, F.; REAL, P. Mensura forestal. San José, Costa Rica: GTZ, IICA. 1997. 586 p.

RICHTER, A. Forsteinrichtung. Leipzing: Neumann Verlag, 1963. 204 p.

ROCHA, R. M.; HIGUCHI, N.; SANTOS, J. dos; NAKAMURA, S.; SILVA, R. P.; PINTO, A. C. M.; TRIBUZY, E. S. Taxas de recrutamento e mortalidade e mudanças de estoques de fitomassa da floresta primária na região de Manaus - AM. In: HIGUCHI, N.; SANTOS, J, dos; SAMPAIO, P. de T.B.; MARENCO, R.A.; FERRAZ, J.; SALES, P.C. de; SAITO, M.; MATSUMOTO, S. (Org.). Projeto Jacaranda, fase II: pesquisas florestais na Amazônia Central, Manaus, AM, 2003. p. 43-54.

ROSSI, L. M. B.; KOEHLER, H. S.; SANQUETTA, C. R. ARCE, J. E. Modelagem de mortalidade em florestas naturais. Floresta, Curitiba, v. 37, n. 2, p. 275-291, 2007.

SANQUETTA, C. R. Fundamentos biométricos dos modelos de simulação florestal. Curitiba: FUPEF, 1996. 49 p. (Série Didática, n. 08).

SCHNEIDER, P. R. Manejo florestal: planejamento da produção florestal. Santa Maria, RS, UFSM, 2009. 613 p.

SCOLFORO, J. R. S. Biometria florestal: modelos de crescimento e produção florestal. Lavras. UFLA/FAEPE, 2006. 393 p.

SHUGGART, H. H. A theory of forest dynamics: the ecological for succession model. New York: Springer-Verlag, 1984. 278 p.

SILVA, J. N. M.; SILVA, S. M. A. da; COSTA, D. H. M.; BAIMA, A. M. V.; OLIVEIRA, L. C. de; CARVALHO, J. O. P. de; LOPES, J. do C. A. Crescimento, mortalidade e recrutamento em florestas de terra firme da Amazônia Oriental: observações nas regiões do Tapajós e Jari. In: SILVA, J. N. M.; CARVALHO, J. O. P. de; YARED, J. A. G. (Ed.). A silvicultura na Amazônia Oriental: contribuições do projeto EMBRAPA/DFID. Belém, PA: Embrapa Amazônia Oriental, 2001. p. 291-308.

SINGH, V. P. On application of the Weibull distribution in hydrology. Water Resources Management, Dordrecht, v. 1, n. 1, p. 33-43, 1987.

SOLOMOM, M. E. Dinâmica de populações. São Paulo: EPU, 1980. $78 \mathrm{p}$. 
SYNNOTT, T. J. A manual of permanent plot procedures for tropical rainforest. Oxford: Commonwealth Forestry Institute; Unit of Tropical Silviculture, 1978. 67 p. (Tropical Forestry paper, n. 14)

TABARELLI, M.; MANTOVANI, W. Colonização de clareiras naturais na floresta Atlântica no Sudeste do Brasil. Revista Brasileira de Botânica, São Paulo, SP, v. 20, n. 1, p. 57-66, 1997.

TSUCHIYA, A.; TANAKA, A.; HIGUCHI, N. LISBOA, P. B. Growth of tree and microclimates in a gap dependent Forest in Central Amazonia. Boletim do Museu Paraense Emílio Goeldi, Belém, PA, v. 1, n. 2, p. 47-63, 2006.

UMANA, C. L. A.; ALENCAR, J. C. Distribuições diamétricas da floresta tropical úmida em uma área no município de Itacoatiara AM. Acta Amazonica, Manaus, v. 28, n. 2, p. 167-190, 1998.

VANCLAY, J. K. Modelling forest growth and yield: applications to mixed tropical forests. Waallingford: CAB International, 1994. 312 p.
WALCK, C. Hand-book on statistical distributions for experimentalists. Stockholm: University of Stockholm, 2007. 190 p.

WEIBULL, W. A statistical theory of the strength of materials. In: TRE ROYAL SWEDISH INSTITUTE FOR ENGINEERING RESEARCH. Proceedings... Stockholm, 1939. v. 151. p. 1-51.

WEIBULL, W. A statistical distribution function of wide applicability. Journal of applied mechanics, Stockholm, v. 73, p. 405-413, 1951.

WRIGHT, S. J.; MUlLER LANDAU, H. C.; CONDIT, R.; HUBBELL, S. Gap-dependent recruitment, realized vital rates, and size distributions of tropical trees. Ecology, Tempe, v. 84, n. 12, p. $3174-3185,2003$.

ZEIDE, B. Analysis of growth equations. Forest Science, Bethesda, v.39, n. 3, p. 594-616, 1993. 
